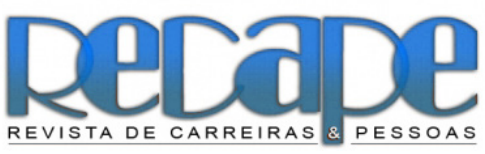

ISSN: 2237-1427

Vol. 10 | No 3 | Ano 2020

Páginas 352-374

\section{Josestenne Bezerra} do Amaral

Pesquisadora da Universidade Federal do Ceará (Brasil) josyamaral1978@gmail.com

Luis Eduardo Brandão Paiva

Pesquisador da Universidade Federal do Ceará (Brasil) edubrandas@gmail.com

\section{Tereza Cristina Batista de Lima}

Professora do Programa de PósGraduação em Administração e Controladoria da Universidade Federal do Ceará (Brasil) tcblima@uol.com.br

Dados para contato:

Luis Eduardo Brandão Paiva Universidade Federal do Ceará Av. da Universidade, 2853 - Benfica, 60020-181, Fortaleza, CE, Brasil.

URL da Homepage:

http://www.ufc.br

Recebido em: 24/09/2019

Aprovado em: 14/05/2020

DOI:

http://dx.doi.org/10.20503/recape. v10i3.45153
CULTURA DE APRENDIZAGEM E DESEMPENHO ORGANIZACIONAL EM UMA EMPRESA PÚBLICA

\author{
LEARNING CULTURE AND ORGANIZATIONAL \\ PERFORMANCE IN A PUBLIC COMPANY
}

CULTURA DE APRENDIZAJE Y DESEMPEÑO

ORGANIZACIONAL EN UNA EMPRESA PÚBLICA

\section{RESUMO}

Este estudo objetivou analisar a influência da cultura de aprendizagem no desempenho organizacional. Trata-se de uma pesquisa quantitativa junto a empregados de uma empresa pública estadual. Baseou-se em Yang (2003), adotando-se o Dimensions of the Learning Organization Questionnaire (Dloq). Para a análise dos dados utilizam-se técnicas multivariadas, como análise fatorial e regressão logística. Os resultados apontam que as dimensões da cultura de aprendizagem que mais influenciam as dimensões do desempenho organizacional são, principalmente, as dimensões Colaboração e aprendizagem em equipe influenciando a dimensão Desempenho do conhecimento e dimensão Delegação de poder e responsabilidade influenciando a dimensão Desempenho financeiro.

Palavras-chave: Cultura Organizacional. Cultura deAprendizagem. Desempenho Organizacional.

\section{ABSTRACT}

This study aimed to analyze the influence of learning culture on organizational performance. This is a quantitative survey of employees of a state-owned company. It was based on Yang (2003), adopting the Dimensions of the Learning Organization Questionnaire (Dloq). Multivariate techniques such as factor analysis and logistic regression are used for data analysis. The results indicate that the dimensions of the learning culture that most influence the dimensions of organizational performance are mainly the dimensions Collaboration and team learning influencing the dimension Knowledge performance and dimension Delegation of power and responsibility influencing the dimension Financial performance.

Keywords: Organizational Culture. Learning Culture. Organizational Performance.

\section{RESUMEN}

Este estudio tuvo como objetivo analizar la influencia de la cultura del aprendizaje en el desempeño organizacional. Esta es una encuesta cuantitativa de empleados de una empresa estatal. Se basó en Yang (2003), adoptando el Cuestionario Dimensiones de la Organización de Aprendizaje (Dloq). Las técnicas multivariantes como el análisis factorial y la regresión 
logística se utilizan para el análisis de datos. Los resultados indican que las dimensiones de la cultura de aprendizaje que más influyen en las dimensiones del desempeño organizacional son principalmente las dimensiones Colaboración y aprendizaje en equipo que influyen en la dimensión Desempeño y dimensión del conocimiento Delegación de poder y responsabilidad que influyen en la dimensión Desempeño financiero.

Palabras clave: Cultura Organizacional. Cultura de Aprendizaje. Desempeño Organizacional.

\section{INTRODUÇÃO}

As grandes transformações e mudanças nas políticas de gestão de pessoas que vem ocorrendo nos últimos anos trazem à tona diversos conceitos e discussões relacionadas a cultura organizacional, que por sua vez, tem um papel crucial no desempenho do processo de gestão, influenciando o sucesso da sua implementação num contexto de alta competitividade entre as organizações (FERNANDES; ALTURAS; LAUREANO, 2015; SCHMITZ et al., 2014). Parte dessas discussões se concentra nas relações entre desempenho organizacional e aprendizagem organizacional. Essas possibilidades podem direcionar as empresas em busca de um desempenho positivo, sejam em termos financeiros, comerciais, de mercado, assim como também o desempenho das pessoas que atuam nos processos das organizações.

Nesse sentido, o cenário mundial sinaliza diversas mudanças no sentido de que as empresas necessitam funcionar e se organizar mais eficazmente no ambiente, em que a competitividade valoriza o diferencial do empregado em cada segmento. Nesse contexto contemporâneo de alta competitividade entre organizações, a aprendizagem organizacional mantida por um ambiente de cultura de aprendizagem é considerada um fator-chave no âmbito gerencial de alavancagem de resultados (SCHMITZ et al., 2014).

Vale ressaltar a perspectiva de Arruda et al. (2015), tendo em vista que os fenômenos da cultura e aprendizagem organizacional passam a ser utilizadas nesse novo contexto, visto que objetivam aprimorar o desempenho e promover o dinamismo e a flexibilidade para tornar que os processos organizacionais possam se tornar mais eficazes e eficientes.

Por meio de uma cultura organizacional, voltada para a aprendizagem, pode-se construir vantagem competitiva e desenvolver de forma mais eficaz o conhecimento e a tecnologia na organização, e isto, consequentemente, contribui para a inovação organizacional (BATES; KHASAWNEH, 2005; OLIVA; SHINYASHIKI, 2016). Dessa forma, identificar as contribuições da cultura de aprendizagem por meio da compreensão de uma organização que aprende e que, portanto, que melhora seu desempenho a partir desse aprendizado, contribui para enfrentar os problemas atuais e desenvolver 
estratégias de recursos humanos voltadas para a eficiência da organização (LAU et al., 2017).

Stewart (2002) afirma que o conhecimento é envolvido pela expertise. Por sua vez, Wood Jr. (2007) apresenta o conceito de conhecimento e expertise, em que expertise é uma fonte de vantagem competitiva e, consequentemente, tem peso determinante nos lucros das empresas. A junção entre o conhecimento e a expertise permite às essas empresas responderem às necessidades dos clientes, criando vantagem competitiva.

O desempenho de uma organização pode ser mensurado por variáveis que consideram assertivas (MARSICK, WATKINS; 2003). Portanto, indicadores e métricas podem ser considerados substanciais que permitem a mensuração do desempenho no contexto organizacional. Sendo assim, Kaplan e Norton (2004) afirmam que, se a organização anseia sobreviver e prosperar na Era do Conhecimento, esta deve se utilizar de um sistema de gestão do desempenho alinhado com sua estratégia.

O envolvimento dos gestores na implementação da estratégia e na geração de desempenho positivo tem uma grande relevância uma vez que estes são a força de trabalho responsável por promover a disseminação e implementação das diretrizes estratégicas oriundas da Alta Administração.

A avaliação da relação entre a cultura de aprendizagem e o desempenho organizacional oferece a possibilidade de verificar as práticas de aprendizagem organizacional e a relação dessas com os resultados proporcionados, levando em conta objetivos estabelecidos no planejamento organizacional (HUNG et al., 2010; LOIOLA; LEOPOLDINO, 2013).

Diante da necessidade de se conhecer possíveis influências existentes entre a cultura de aprendizagem e o desempenho organizacional em virtude da importância do tema e da escassez de discussão sobre esta temática no setor público, a questão norteadora desta pesquisa é: Qual a influência existente entre a cultura de aprendizagem e o desempenho organizacional em uma empresa pública?

Nica (2013) enfatiza a pertinência de estudar o contexto da investigação empírica dos funcionários do setor público, considerando, sobretudo, fenômenos relacionados à cultura organizacional. É pertinente também considerar que estudos empíricos têm encontrado relações positivas entre cultura de aprendizagem e desempenho organizacional (MARSICK; WATKINS, 2003).

Dessa forma, esta pesquisa tem como objetivo analisar a influência da cultura de aprendizagem no desempenho organizacional, além de fornecer elementos que orientem as organizações no que se refere aos fatores que afetam o desempenho organizacional.

Para o desempenho organizacional, utiliza-se a ótica da teórica do desempenho financeiro e do desempenho do conhecimento, e isto se alinha à perspectiva de Marsick e Watkins (2003), o que 
confere um caráter bidimensional para o contexto do desempenho organizacional.

Quanto à relevância gerencial do estudo, espera-se que os resultados desta pesquisa possam colaborar na formulação de políticas para o envolvimento dos gestores na implementação da estratégia e na geração de desempenho positivo o qual tem uma grande relevância uma vez que estes são a força de trabalho responsável por promover a disseminação e implementação das diretrizes estratégicas oriundas da Alta Administração. Considera-se, sobretudo, a perspectiva de Jung et al. (2009), de que considera que a cultura organizacional é primordial para o gerenciamento organizacional.

Academicamente, este estudo torna-se fundamental por suplantar a lacuna da literatura que permite analisar a influência do desempenho organizacional - por meio do desempenho do conhecimento e financeiro - na cultura de aprendizagem, tomando-se como investigação empírica funcionários do setor público. Este estudo reflete, ainda, em contribuições para futuros estudos em relação aos temas em questão, além de orientar as organizações no que se refere aos fatores que podem afetar o seu desempenho organizacional.

Serão discutidos, a seguir, os elementos teóricos que fundamentaram a pesquisa, no que se refere à cultura de aprendizagem e ao desempenho organizacional, a metodologia de pesquisa, análise e discussão dos dados, além das considerações finais e referências.

\section{FUNDAMENTAÇÃO TEÓRICA}

\subsection{Cultura organizacional e de aprendizagem}

O estudo da cultura organizacional ganhou força nos anos de 1980, a partir do desempenho observado nas empresas japonesas e do envolvimento dos trabalhadores com os valores e a filosofia da empresa (FERREIRA; ASSMAR, 2008). Nessa época, a cultura organizacional se converteu em assunto prioritário nos estudos de comportamento organizacional (FORTADO; FADIL, 2012; DIAS, 2013).

As organizações existem num meio, constituído pela cultura e estrutura social da sociedade. Para se obter eficiência numa organização, é necessário não só que seus empregados aprendam os métodos de trabalho, mas também compreendam a missão da organização, a sua forma de realizar as coisas, o seu clima ou cultura (HOGAN; COOTE, 2014; SCHEIN, 2009).

O termo cultura deriva metaforicamente da ideia de cultivo, ou seja, o processo de preparar a terra. Essa palavra refere-se ao padrão de desenvolvimento decorrente do sistema de conhecimento, ideologia, valores, leis e rituais diários de uma sociedade (MORGAN, 2007).

A cultura organizacional pode ser compreendida como um padrão compartilhado de crenças e 
valores que ajudam os indivíduos a entender o funcionamento organizacional e também provê a eles normas para o comportamento na organização (DESHPANDE; WEBSTER, 1989). A cultura cumpre diversos papeis na organização. Ela é a argamassa social que ajuda a manter a organização coesa fornecendo os padrões adequados para fundamentar o que os funcionários vão fazer ou dizer (ROBBINS, 2009).

Como Schein (2009) apontou, a cultura organizacional fornece um sistema de expectativas que estabelece normas e um padrão de comportamento para os funcionários, fornecendo uma razão para o comportamento de liderança. Os líderes devem compreender profundamente a cultura organizacional para comunicar e implementar novas visões e inspirar o compromisso do seguidor com a visão. Quanto mais forte for a cultura organizacional, mais forte será o impacto na eficácia da organização (SCHEIN, 2009).

Embora as forças do mercado, o posicionamento competitivo, a estratégia e a tecnologia sejam evidentemente importantes, empresas altamente bem-sucedidas capitalizaram o valor que reside no desenvolvimento e gerenciamento de uma cultura organizacional única (AZANZA; MORIANO; MOLERO, 2013).

Para Arruda et al. (2015), a cultura de uma organização está diretamente relacionada aos aspectos que envolvem a aprendizagem organizacional. Dessa forma, os elementos da cultura fornecem o suporte necessário para que os processos de aprendizagem ocorram nas organizações. Uma vez compartilhados, esses elementos permitem que os membros de um grupo comunguem com os mesmos ideais e filosofia de pensamento, e por meio da interação social, promovam a interseção da aprendizagem com a cultura organizacional.

No âmago da concepção de cultura organizacional é que o processo de aprendizagem se origina, seja no momento da sua constituição, como na transmissão dos valores básicos aos novos colaboradores de uma organização (ARRUDA et al., 2015; MARANHÃO; DE SÁ, 2019; RIJAL, 2016). Senge (2006) sugere que as organizações só aprendem por meio de indivíduos que aprendem. A aprendizagem individual não garante a aprendizagem organizacional. Entretanto, sem ela, a aprendizagem organizacional não ocorre.

Ao tratarem da aprendizagem no nível individual e organizacional, Marsick e Watkins (2003) entendem que, no primeiro caso, a aprendizagem ocorre quando os indivíduos se deparam com surpresas ou desafios que servem de estímulos para uma reação. Já a aprendizagem organizacional é o resultado de um processo interativo e interdependente. Nesse caso, os estímulos podem vir de uma nova norma, um novo concorrente, mudanças no mercado, novas tecnologias, insatisfação por parte de clientes, novas demandas e exigências ou outra mudança qualquer que altere o status quo da organização. Ademais, o entendimento do contexto ambiental da organização, tanto interno quanto 
externo, pode auxiliar a organização a agir de forma proativa.

Na perspectiva de Schein (2009), a única forma de construir uma cultura de aprendizagem que continue aprendendo é os próprios líderes aprenderem a perceber que há muita coisa que não sabem. Além disso, eles devem ensinar os outros a aceitarem suas limitações. A aprendizagem relaciona-se continuamente com o desempenho organizacional (BATES; KHASAWNEH, 2005). Nesse contexto, a tarefa da aprendizagem se torna uma responsabilidade compartilhada dos indivíduos, em que pode estar alinhada ao desempenho organizacional.

\subsection{Desempenho organizacional}

Medidas de desempenho emitem sinais vitais da organização: comunicam a estratégia para baixo, os resultados dos processos para cima e o controle dos processos, interligando estratégias, recursos e processos. Medição de desempenho compreende quantificação da relação entre processos, saídas e metas, de modo contínuo, o que, por feedback, leva ao estabelecimento de novas metas e ao ajuste da estratégia. De fato, se não for possível medir, não se pode controlar; se não se controlar, não se pode gerenciar; se não se gerenciar, não se pode melhorar (BRANDÃO; BORGES-ANDRADE; GUIMARÃES, 2012; DELANEY; HUSELID, 1996; RAFAELI; MULLER, 2007).

Conforme destacam Kaplan e Norton (2004), as empresas estão abandonando os modelos de gerenciamento de desempenho que se baseiam apenas em critérios financeiros pois estes não captam toda a dinâmica do desempenho empresarial no atual contexto competitivo baseado no conhecimento. Propõem o modelo do Balanced Scorecard, no qual existe a perspectiva de aprendizagem e crescimento que tem como objetivo desenvolver o ativo intangível da organização, por meio da implementação de métodos e técnicas de gestão que sejam capazes de desenvolver e preservar o seu capital intelectual.

Segundo Arruda et al. (2015), essa perspectiva impulsiona melhorias nos processos internos da organização, que, por sua vez, exercem o máximo de alavancagem no fornecimento de valor para os clientes, contribuindo assim para a melhoria do resultado financeiro.

Marsick e Watkins (2003), ao estudarem a aprendizagem em organizações, identificaram, em seus estudos, que uma cultura orientada para apoiar a aprendizagem pode levar ao melhor desempenho. O desempenho organizacional sofre, portanto, forte influência da cultura, uma vez que esta envolve também o comportamento de seus membros e os diversos setores e níveis das empresas.

Fiol e Lyles (1985) apontam que a cultura consiste em compartilhar crenças, ideologias e normas que refletem as ações da organização. Assim, sugerem que a cultura pode ser considerada um preditor de ações organizacionais que podem influenciar o desempenho. 
A relação entre a cultura de aprendizagem e o desempenho organizacional, foco desta pesquisa, são explorados a seguir.

\subsection{Cultura de aprendizagem e desempenho organizacional}

A principal relação entre a aprendizagem com o desempenho organizacional está na capacidade do indivíduo. O processo de aprendizagem está relacionado com o desempenho organizacional. O desempenho varia de acordo com a empresa e está, por sua vez, relacionado com o aprendizado dos indivíduos (RICHARD et al., 2009), com o intuito de que estes possam tomar decisões (BRITO; VASCONCELOS, 2005).

Segundo Vargas-Hernandéz et al. (2016), os valores, hábitos e tradições cultivadas ao longo do tempo em uma organização, definem a forma como as pessoas se comportam o que contribui para a aprendizagem dos membros desta organização.

Com o objetivo de mensurar a relação existente entre cultura de aprendizagem e desempenho organizacional Marsick e Watkins (2003) desenvolveram a escala Dimensions of the Learning Organization Questionnaire (Dloq). Yang (2003) propôs um modelo resumido desta escala, o Dloq-A. O quadro1 a seguir, apresenta os fatores desta escala no que se refere a cultura de aprendizagem nas organizações.

Quadro 1 - Definições dos fatores de escala de cultura de aprendizagem nas organizações

\begin{tabular}{|c|c|}
\hline Fator & Definição \\
\hline \multicolumn{2}{|c|}{ I - Fatores relacionados com a cultura de aprendizagem nas organizações } \\
\hline $\begin{array}{l}1 \text { Oportunidades para a } \\
\text { aprendizagem contínua }\end{array}$ & $\begin{array}{l}\text { A aprendizagem é incorporada ao trabalho para que as pessoas possam aprender } \\
\text { no local de trabalho. Existem oportunidades para educação e crescimento } \\
\text { permanentes. }\end{array}$ \\
\hline $\begin{array}{l}2 \text { Questionamento e } \\
\text { diálogo }\end{array}$ & $\begin{array}{l}\text { As pessoas desenvolvem habilidades de raciocínio produtivo para expressar seus } \\
\text { pontos de vista e capacidade para ouvir e perguntar a opinião dos outros; a cultura } \\
\text { é modificada para apoiar o questionamento, o feedback e a experimentação. }\end{array}$ \\
\hline $\begin{array}{l}3 \text { Colaboração e } \\
\text { aprendizagem em equipe }\end{array}$ & $\begin{array}{l}\text { O trabalho é criado para usar grupos para acessar diferentes modos de pensar; } \\
\text { espera-se que os grupos trabalhem e aprendam juntos; a colaboração é valorizada } \\
\text { pela cultura e recompensada. }\end{array}$ \\
\hline $\begin{array}{l}4 \text { Sistemas para capturar e } \\
\text { compartilhar a } \\
\text { aprendizagem }\end{array}$ & $\begin{array}{l}\text { Sistemas de alta e baixa tecnologia para compartilhar a aprendizagem são criados e } \\
\text { integrados com o trabalho; há acesso; os sistemas são mantidos. }\end{array}$ \\
\hline $\begin{array}{l}5 \text { Delegação de poder e } \\
\text { responsabilidade }\end{array}$ & $\begin{array}{l}\text { As pessoas são envolvidas em estabelecer, ter e implementar uma visão coletiva; a } \\
\text { responsabilidade é distribuída na hora de tomar decisões para motivar as pessoas a } \\
\text { aprender aquilo que será de sua responsabilidade. }\end{array}$ \\
\hline $\begin{array}{l}6 \text { Desenvolvimento da visão } \\
\text { sistêmica da organização }\end{array}$ & $\begin{array}{l}\text { As pessoas são ajudadas a ver o efeito de seu trabalho em toda a empresa; } \\
\text { examinam o ambiente e usam as informações para ajustar as práticas do trabalho; } \\
\text { a organização é ligada às suas comunidades. }\end{array}$ \\
\hline
\end{tabular}




\begin{tabular}{|l|l|}
\hline $\begin{array}{l}\text { 7 Estímulo à liderança } \\
\text { estratégica para a } \\
\text { aprendizagem }\end{array}$ & $\begin{array}{l}\text { Os líderes modelam, prestigiam e apoiam a aprendizagem; a liderança utiliza a } \\
\text { aprendizagem estrategicamente para obter resultados nos negócios. }\end{array}$ \\
\hline II - Fatores relacionados com o desempenho organizacional \\
\hline 1 Desempenho financeiro & Estado de saúde financeira e recursos disponíveis para crescimento. \\
\hline $\begin{array}{l}2 \text { Ganho de conhecimento } \\
\text { organizacional }\end{array}$ & $\begin{array}{l}\text { Melhora nos produtos e serviços por causa de aprendizagem e capacidade de } \\
\text { conhecimento (principais indicadores de capital intelectual). }\end{array}$ \\
\hline
\end{tabular}

Fonte: Adaptado de Marsick e Watkins (2003).

Tomando-se como base o Quadro 1, Marsick e Watkins (2003) relacionam os fatores/dimensões da cultura de aprendizagem e do desempenho organizacional, enfatizando, sobretudo, as suas definições. A seguir, destaca-se a metodologia da pesquisa com foco nos fatores adotados na escala de cultura de aprendizagem e no desempenho organizacional.

\section{PROCEDIMENTOS METODOLÓGICOS}

Este estudo pode ser classificado como uma pesquisa quantitativa, de natureza descritiva (COLLIS; HUSSEY, 2005). Os dados para a presente pesquisa foram coletados no mês de março de 2017, por meio de uma survey, que segundo Babbie (2005), é um método lógico, determinístico, generalista, parcimonioso e específico. A survey possibilita identificar as fontes de eventos, elaborar clara e rigorosamente um modelo lógico, examinar criteriosamente a importância relativa de cada variável e obter correlações e resultados corretos.

O universo da pesquisa compreendeu os empregados próprios de uma empresa pública estadual, que tinham acesso a rede de computadores em seu ambiente de trabalho, perfazendo um total de 1035 empregados. A amostra foi definida por meio de amostragem aleatória simples, composta por 388 indivíduos.

A empresa ALFA (nome fictício), objeto deste estudo, esteve presente no ranking das Melhores Empresas para se Trabalhar da Revista Você S/A, em 2015, sendo considerada a $3^{a}$ melhor empresa pública do Brasil. Além disso, vale destacar que a empresa já está a 45 anos no mercado de saneamento básico, estando entre uma das 5 maiores empresas no seu setor de atuação sendo o Sistema Nacional de Informações de Saneamento (SNIS, 2015).

Neste estudo, utilizou-se a versão simplificada do instrumento Dimensions of the Learning Organization Questionnaire (Dloq), desenvolvida por Yang (2003), nomeado pela autora de Dloq-A 
e validada no Brasil por Menezes, Guimarães e Bido (2011). Essa versão é composta de 27 itens distribuídos nas sete dimensões da cultura de aprendizagem - oportunidades para a aprendizagem contínua, questionamento e diálogo, colaboração e aprendizagem em equipe, sistemas para capturar e compartilhar a aprendizagem, delegação de poder e responsabilidade, desenvolvimento da visão sistêmica da organização e estímulo à liderança estratégica para a aprendizagem - e nas duas dimensões do desempenho organizacional - desempenho financeiro e ganho de conhecimento organizacional.

O questionário, instrumento da pesquisa, teve a sua estruturação mediante um conjunto de itens preestabelecidos, constituídos em uma escala likert de 6 pontos, variando entre 1 "nunca" e 6 "sempre". Os questionários foram enviados por meio de um link para todos os empregados que tinham e-mail institucional de forma que os respondentes preenchessem os questionários diretamente em meio digital.

Para análise dos dados, adotou-se a estatística descritiva para caracterizar o perfil da amostra. Em seguida, empregou-se a estatística multivariada, como a análise fatorial exploratória - para verificar as cargas fatoriais das variáveis, utilizando, portanto, o critério da variável substituta para cada fator, em que se enfatiza a variável com maior carga fatorial, visto que é a que tem maior poder de explicação no fator (HAIR et al., 2009).

Logo após, calculou-se o alfa de Cronbach - com o intuito de verificar a confiabilidade interna da escala composta pelas dimensões dos construtos "cultura de aprendizagem" e "desempenho organizacional". Geralmente, afirma-se um valor aceitável de 0,7; valores abaixo disso podem dizer que a escala não é confiável (FIELD, 2009).

Para finalizar as técnicas de análise estatística dos dados, fez-se o uso de modelos de regressão logística, a qual tem como objetivo “(...) usar as variáveis independentes cujos valores são conhecidos para prever os valores da variável dependente escolhida pelo pesquisador" (HAIR et al., 2009, p. 154).

As análises estatísticas das respostas tiveram tratamento de dados com o auxílio do software Statistical Package for the Social Sciences (SPSS) versão 21.0.

\section{ANÁLISE E DISCUSSÃO DOS RESULTADOS}

Com a finalidade de discutir os resultados obtidos na pesquisa, serão abordadas, a seguir, a caracterização da amostra da pesquisa, a análise multivariada dos dados, envolvendo a consistência interna e a análise fatorial exploratória, bem como os resultados do modelo de regressão logística. 


\subsection{Caracterização da amostra}

Para uma primeira análise, destacam-se as variáveis demográficas consideradas no estudo. A amostra da pesquisa é composta por 388 indivíduos, denominados de empregados próprios, sendo que 217 é representado por homens (55,9\%) e 171 por mulheres (44,1\%). A maioria dos pesquisados apresenta nível de especialização $(35,6 \%)$ e, logo em seguida, graduação (27,8\%). A idade média da amostra é de 42 anos, com desvio-padrão de 11,667; variando entre a mínima de 20 e a máxima de 71 anos.

No que concerne a lotação dos indivíduos, se estavam na capital ou no interior, enfatiza-se que $67 \%$ concentra-se na capital, enquanto $33 \%$ concentra-se no interior do Estado.

No estudo de Arruda et al. (2015), constatou-se o perfil da amostra pesquisa era composta por mulheres, jovens e com pouco tempo de empresa, diferentemente do perfil da empresa do presente estudo. Pode-se supor que essa diferença seja por conta do tempo em que a empresa pesquisada tem de existência e, principalmente por caracterizar-se como uma empresa pública, bem como pelo fato dos pesquisados serem funcionários públicos, o que faz com que estes tenham uma média de idade superior ao perfil de respondentes dos estudos de Arruda et al. (2015).

\subsection{Consistência interna}

A consistência interna do Dimensions of the Learning Organization Questionnaire (Dloq) foi avaliada pela consistência interna de fidedignidade do alfa de cronbach. Os valores obtidos no coeficiente do Cronbach para as dimensões da cultura de aprendizagem e do desempenho organizacional permitiram atestar consistência interna satisfatória, visto que todos os valores foram acima de 0,7 (HAIR et al., 2009), e isto é pertinente para indicar a consistência interna dos componentes, o que reflete na fidedignidade aceitável.

Além do alfa de cronbach, mostram-se a quantidade de itens de cada dimensão, média o mínimo e máximo de cada dimensão da cultura de aprendizagem (TABELA 1).

Tabela 1 - Dimensões da cultura de aprendizagem

\begin{tabular}{|l|c|c|c|c|c|}
\hline Dimensões & Alfa de cronbach & $N^{\circ}$ de itens & Média & Mínimo & Máximo \\
\hline $\begin{array}{l}\text { Oportunidades para Aprendizagem } \\
\text { Contínua }\end{array}$ & 0,721 & 3 & 4,113 & 3,724 & 4,338 \\
\hline Questionamento e Diálogo & 0,826 & 3 & 3,646 & 3,526 & 3,755 \\
\hline
\end{tabular}




\begin{tabular}{|l|c|c|c|c|c|}
\hline Colaboração e Aprendizagem em Equipe & 0,860 & 3 & 3,924 & 3,724 & 4,129 \\
\hline $\begin{array}{l}\text { Sistemas para Capturar e Compartilhar a } \\
\text { Aprendizagem }\end{array}$ & 0,829 & 3 & 3,820 & 3,438 & 4,343 \\
\hline Deleção de Poder e Responsabilidade & 0,878 & 3 & 3,622 & 3,528 & 3,698 \\
\hline $\begin{array}{l}\text { Desenvolvimento da Visão Sistêmica da } \\
\text { Organização }\end{array}$ & 0,890 & 3 & 4,043 & 3,905 & 4,170 \\
\hline $\begin{array}{l}\text { Estímulo à Liderança Estratégica para a } \\
\text { Aprendizagem }\end{array}$ & 0,913 & 3 & 3,893 & 3,750 & 4,003 \\
\hline
\end{tabular}

Fonte: Dados da pesquisa.

Dentre as sete dimensões do DLOQ-A, as que apresentaram maiores médias foram as dimensões "Oportunidades para a aprendizagem contínua" e "Desenvolvimento da visão sistêmica da organização". As médias para essas dimensões foram 4,113 e 4,043, respectivamente. Ademais, vale-se enfatizar os maiores valores do alfa de cronbach $(\alpha)$ : "Estímulo à liderança estratégica para a aprendizagem" com valor do a de 0,913; e "Oportunidades para a aprendizagem contínua" com o valor do a de 0,890 .

Os dois menores valores do cronbach ( $\alpha$ ) foram as dimensões: "Oportunidades para aprendizagem contínua", com valor do a de 0,721 e "Sistemas para capturar e compartilhar a aprendizagem, com valor do a de 0,829.

O estudo de Queiroz et al. (2014), corroboram a esses resultados quando demonstram que na organização a aprendizagem é incorporada ao trabalho e que existem, por sua vez, oportunidades para educação e crescimento dos indivíduos. Além disso, as pessoas são envolvidas em estabelecer, ter e implementar uma visão coletiva.

Pela Tabela 2 verifica-se o alfa de cronbach ( $\alpha$ ), média o mínimo e máximo de cada dimensão do desempenho organizacional.

Tabela 2 - Dimensões do desempenho organizacional

\begin{tabular}{|l|c|c|c|c|c|}
\hline Dimensões & Alfa de Cronbach & $\begin{array}{c}\text { No de } \\
\text { itens }\end{array}$ & Média & Mínimo & Máximo \\
\hline Desempenho financeiro & 0,850 & 3 & 3,914 & 3,750 & 4,015 \\
Desempenho do conhecimento organizacional & 0,898 & 3 & 3,858 & 3,776 & 3,902 \\
\hline
\end{tabular}

Fonte: Dados da pesquisa. 
Quando analisadas as médias das dimensões do desempenho organizacional, conforme tabela 2, observa-se que a maior média foi da dimensão "Desempenho financeiro", com valor de 3,914. Essa dimensão apresentou valor do alfa de $\operatorname{cronbach}(\alpha)$ de 0,850 . Esse resultado sugere que os indivíduos pesquisados percebem melhor o estado de saúde financeira e recursos disponíveis para crescimento da organização. Em relação à dimensão "Desempenho do conhecimento organizacional", a mesma também apresenta uma média alta tanto comparando com a dimensão anterior, como comparando o resultado com o valor máximo dessa dimensão de 3,902 versus 4,015.

\subsection{Análise fatorial exploratória}

A partir da análise fatorial para os fatores relacionados à aprendizagem nas organizações e ao desempenho organizacional, pode-se evidenciar os resultados para cada variável, o que se vale por extrair a maior carga fatorial, ou seja, o autovalor de cada fator. Essa variável com maior valor é a que apresenta maior representatividade e poder de explicação dentro de cada fator. Portanto, observa-se pela Tabela 3 as cargas fatoriais, média e desvio padrão das variáveis dentro de cada fator/dimensão.

Tabela 3 - Análise fatorial para cultura de aprendizagem

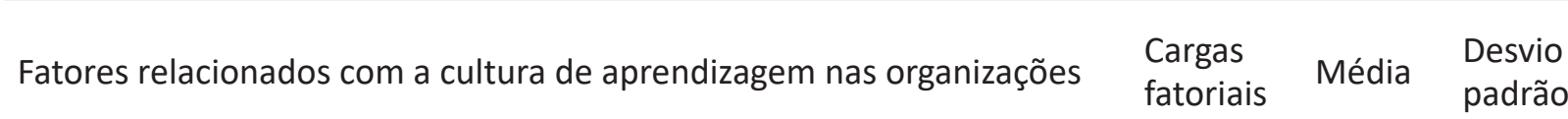

Oportunidades

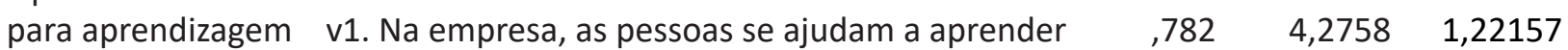

continua

v2. A empresa libera o funcionário para aprender

$, 812 \quad 4,3376 \quad 1,39838$

v3. As pessoas são recompensadas quando aprendem

,809 $3,7242 \quad 1,43010$

Questionamento e diálogo

v4. As pessoas dão feedback aberto umas às outras

,852 $\quad 3,5258 \quad 1,29074$

v5. Na empresa, quando as pessoas dão sua opinião, também perguntam o que os outros pensam

$, 861 \quad 3,6572 \quad 1,23620$

v6. As pessoas desenvolvem confiança nos outros 


\begin{tabular}{|c|c|c|c|c|}
\hline \multirow[t]{3}{*}{$\begin{array}{l}\text { Colaboração e } \\
\text { aprendizagem em } \\
\text { equipe }\end{array}$} & $\begin{array}{l}\text { v7. Equipes e grupos de trabalho da empresa têm } \\
\text { liberdade para discutir suas metas conforme as } \\
\text { suas necessidades }\end{array}$ & 881 & 4,1289 & 1,33096 \\
\hline & $\begin{array}{l}\text { v8. As equipes avaliam sua conduta com base nos } \\
\text { resultados das discussões em grupo ou nos dados } \\
\text { coletados }\end{array}$ & 906 & 3,9201 & 1,21630 \\
\hline & $\begin{array}{l}\text { v9. As equipes acreditam que a empresa seguirá } \\
\text { suas recomendações }\end{array}$ & ,864 & 3,7242 & 1,25701 \\
\hline \multirow{3}{*}{$\begin{array}{l}\text { Sistemas para } \\
\text { capturar e } \\
\text { compartilhar a } \\
\text { aprendizagem }\end{array}$} & $\begin{array}{l}\text { v10. A empresa usa sistemas para medir a diferença } \\
\text { entre o desempenho atual e o desempenho } \\
\text { esperado }\end{array}$ & 827 & 4,3428 & 1,34626 \\
\hline & $\begin{array}{l}\text { v11. A empresa disponibiliza o que aprendeu com } \\
\text { os erros para todos os funcionários }\end{array}$ & ,893 & 3,4381 & 1,35973 \\
\hline & $\begin{array}{l}\text { v12. A empresa mede os resultados do tempo e } \\
\text { investimento usados para treinamento }\end{array}$ & 870 & 3,6804 & 1,43451 \\
\hline \multirow[t]{3}{*}{$\begin{array}{l}\text { Delegação } \\
\text { de poder e } \\
\text { responsabilidade }\end{array}$} & $\begin{array}{l}\text { v13. A empresa reconhece as pessoas por suas } \\
\text { iniciativas }\end{array}$ & 890 & 3,6985 & 1,36183 \\
\hline & $\begin{array}{l}\text { v14. A empresa permite as pessoas o controle } \\
\text { sobre os recursos de que precisam para realizar } \\
\text { seu trabalho }\end{array}$ & 910 & 3,6392 & 1,36917 \\
\hline & $\begin{array}{l}\text { v15. A empresa apoia as pessoas que assumem } \\
\text { riscos calculados }\end{array}$ & 889 & 3,5284 & 1,31841 \\
\hline \multirow[t]{3}{*}{$\begin{array}{l}\text { Desenvolvimento } \\
\text { da visão sistêmica } \\
\text { da organização }\end{array}$} & $\begin{array}{l}\text { v16. A empresa encoraja as pessoas a pensar com } \\
\text { uma perspectiva global }\end{array}$ & 905 & 3,9046 & 1,35398 \\
\hline & $\begin{array}{l}\text { v17. A empresa trabalha em conjunto com a } \\
\text { comunidade externa para atender às necessidades } \\
\text { mútuas }\end{array}$ & 907 & 4,0541 & 1,28383 \\
\hline & $\begin{array}{l}\text { v18. As pessoas são estimuladas a obter respostas } \\
\text { de outras partes da empresa quando precisam } \\
\text { resolver problemas }\end{array}$ & 904 & 4,1701 & 1,26038 \\
\hline \multirow{3}{*}{$\begin{array}{l}\text { Estímulo à } \\
\text { liderança } \\
\text { estratégica para a } \\
\text { aprendizagem }\end{array}$} & $\begin{array}{l}\text { v19. Os gerentes orientam e treinam seus } \\
\text { subordinados }\end{array}$ & ,925 & 3,7500 & 1,43844 \\
\hline & $\begin{array}{l}\text { v20. Os gerentes procuram constantemente } \\
\text { oportunidade de aprendizagem }\end{array}$ & ,926 & 3,9253 & 1,40581 \\
\hline & $\begin{array}{l}\text { v21. Os gerentes garantem que as ações } \\
\text { organizacionais são consistentes com os valores da } \\
\text { empresa }\end{array}$ & 917 & 4,0026 & 1,32263 \\
\hline
\end{tabular}

Fonte: Dados da pesquisa.

Utiliza-se o critério da variável substituta - as variáveis das dimensões da cultura de aprendi- 
zagem que obtiveram cargas fatoriais mais elevadas, ou seja, as que apresentam maior explicação dentro de cada dimensão. Ademais, as maiores médias foram a v1. "Na empresa, as pessoas se ajudam a aprender"; v2. "A empresa libera o funcionário para aprender" e a v10. "A empresa usa sistemas para medir a diferença entre o desempenho atual e o desempenho esperado". As médias dessas variáveis foram 4,2758, 4,3376 e 4,3428, respectivamente.

Enquanto isso, as variáveis das dimensões da cultura de aprendizagem que apresentaram as menores médias foram a v4. "As pessoas dão feedback aberto e honesto umas às outras", a v11. "A empresa disponibiliza o que aprendeu com os erros para todos os funcionários" e a v15. "A empresa apoia as pessoas que assumem riscos calculados". As médias dessas variáveis foram 3,5258 , 3,4381 e 3,5284, respectivamente.

Ao analisar a representatividade de cada variável por dimensão, pode-se observar que, com relação à dimensão "Oportunidades para a aprendizagem contínua", a variável que apresenta maior carga fatorial é a v2. "A empresa libera o funcionário para aprender", e isto permite inferir que esta variável é a que melhor representa esta dimensão. Isso significa que, na percepção dos indivíduos, o fato da empresa liberar seus colaboradores para aprender, pode demonstrar que a organização oferece oportunidades para o aprendizado contínuo.

Para a dimensão "Questionamento e diálogo", a variável que melhor representa esta dimensão é a v6. "As pessoas desenvolvem confiança nos outros". Ou seja, a percepção dos respondentes é que a medida em que as pessoas desenvolvem confiança nos outros membros do grupo/organização, sendo assim, este fato melhor configura esta dimensão.

Tomando como base a dimensão "Colaboração e aprendizagem em equipe", a variável mais representatitva para esta dimensão é a v8. "As equipes avaliam sua conduta com base nos resultados das discussões em grupo ou nos dados coletados". Esse resultado demonstra que há colaboração e aprendizagem em equipe quando existe uma avaliação com base em resultados.

A variável v11. "A empresa disponibiliza o que aprendeu com os erros para todos os funcionários" é a mais representativa para a dimensão "Sistemas para capturar e compartilhar a aprendizagem". Para esta dimensão, a medida em que a empresa disponibiliza a todos as lições aprendidas, melhor se configura os sistemas de captura e compartilhamento da aprendizagem.

Para a dimensão "Delegação de poder e responsabilidade", a variável que melhor representa esta dimensão é a v14. "A empresa permite as pessoas o controle sobre os recursos de que precisam para realizar seu trabalho". Nessa dimensão, a percepção é que ela existe quando a empresa permite o controle dos recursos por parte dos seus membros.

Com relação a dimensão "Desenvolvimento da visão sistêmica da organização", a variável que 
obteve maior carga fatorial foi a v17. "A empresa trabalha em conjunto com a comunidade externa para atender às necessidades mútuas". Esse resultado evidencia que os indivíduos percebem essa dimensão quando há um trabalho conjunto entre empresa e meio ambiente.

A variável v20. "Os gerentes procuram constantemente oportunidade de aprendizagem", foi a que apresentou maior carga fatorial na dimensão "Estímulo à liderança estratégica para a aprendizagem" e, portanto, a que melhor representa esta dimensão. Aqui, a percepção é que essa dimensão é representada quando há uma constante procura por oportunidades de aprendizagem por parte dos líderes.

Além dessa primeira análise fatorial para a cultura de aprendizagem, realiza-se também, a seguir, a análise fatorial para o desempenho organizaional, o qual está subdividido nas dimensões "Desempenho financeiro" e "Desempenho do conhecimento organizacional", conforme pode vizualizar pela Tabela 4.

Tabela 4 - Análise fatorial para desempenho organizacional

\begin{tabular}{|c|c|c|c|c|}
\hline \multicolumn{2}{|c|}{ Fatores relacionados ao desempenho organizacional } & \multirow{2}{*}{$\begin{array}{l}\text { Cargas } \\
\text { fatoriais } \\
\text {,867 }\end{array}$} & \multirow{2}{*}{$\begin{array}{l}\text { Média } \\
\text { 3,9768 }\end{array}$} & \multirow{2}{*}{$\begin{array}{l}\text { Desvio } \\
\text { padrão }\end{array}$} \\
\hline \multirow{3}{*}{$\begin{array}{l}\text { Desempenho } \\
\text { financeiro }\end{array}$} & $\begin{array}{l}\text { v22. A produtividade média por empregado é } \\
\text { maior que no ano passado }\end{array}$ & & & \\
\hline & $\begin{array}{l}\text { v23. O tempo para colocar no mercado os } \\
\text { produtos e serviços é menor que no ano passado }\end{array}$ & 904 & 3,7500 & 1,25831 \\
\hline & $\begin{array}{l}\text { v24. O tempo de resposta para lidar com } \\
\text { reclamações dos clientes externos é menor que no } \\
\text { ano passado }\end{array}$ & ,860 & 4,0155 & 1,30186 \\
\hline \multirow{3}{*}{$\begin{array}{l}\text { Desempenho do } \\
\text { conhecimento } \\
\text { organizacional }\end{array}$} & $\begin{array}{l}\text { v25. A satisfação do cliente é maior que no ano } \\
\text { passado }\end{array}$ & 906 & 3,8969 & 1,26764 \\
\hline & $\begin{array}{l}\text { v26. O número de sugestões implementadas pela } \\
\text { empresa é maior que no ano passado }\end{array}$ & ,936 & 3,9021 & 1,30223 \\
\hline & $\begin{array}{l}\text { v27. Na empresa, o número de indivíduos que } \\
\text { aprendem novas habilidades é maior que no ano } \\
\text { passado }\end{array}$ & 894 & 3,7758 & 1,34634 \\
\hline
\end{tabular}

Fonte: Dados da pesquisa.

Para a dimensão "Desempenho financeiro", a variável que apresentou a maior carga fatorial e, portanto, a que melhor representa esta dimensão foi a v23. "O tempo para colocar no mercado os produtos e serviços é menor que no ano passado", ou seja, essa dimensão é percebida quanto 
menor for o tempo necessário para dispor os produtos e serviços no mercado.

Considerando a dimensão "Desempenho do conhecimento organizacional", a variável que apresentou a maior carga fatorial e, portanto, a que melhor representa esta dimensão foi a v26. "O número de sugestões implementadas pela empresa é maior que no ano passado". Essa dimensão é percebida pelos indivíduos quando o número de sugestões implementadas aumenta ao longo do tempo.

O critério da variável substituta é pertinente por permitir, a partir das cargas fatoriais das variáveis mais representativas dentro de cada fator, desenvolver modelos de regressão. Para este estudo, pretende-se, portanto, analisar a influência da cultura de aprendizagem no desempenho organizacional.

\subsection{Resultados do modelo de regressão logística}

A regressão logística verifica a relação entre uma variável dependente em função das variáveis independentes (HAIR et al., 2009). No caso desta pesquisa, a variável dependente é o desempenho organizacional, que será subdividido em dois modelos de regressão: (i) direcionado ao desempenho do conhecimento organizacional; e (ii) desempenho financeiro. Enquanto que as variáveis independentes são as variáveis da cultura de aprendizagem e as variáveis sociodemográficas.

Quanto menor o sig. (grau de significância) na regressão logística, maior o poder de relação da variável independente na variável dependente. Dessa forma, leva-se em consideração a perspectiva de Hair et al. (2009), sugerindo significância menor do que 0,05. Portanto, são consideradas como variáveis de influência no desempenho financeiro as variáveis destacadas, conforme verifica-se pela Tabela 5.

Tabela 5 - Modelo de regressão (variável dependente: desempenho financeiro)

\begin{tabular}{|l|c|c|c|}
\hline \multicolumn{1}{|c|}{ Variáveis } & B & Sig. & Exp(B) \\
\hline Sexo (Masculino) &,- 096 &, 733 &, 908 \\
\hline Escolaridade (Médio/Técnico) & 1,408 &, 483 & 4,089 \\
\hline Escolaridade (Graduação) & 1,831 &, 357 & 6,239 \\
\hline Escolaridade (Especialização) & 1,096 &, 578 & 2,993 \\
\hline Escolaridade (Mestrado) &, 760 &, 703 & 2,139 \\
\hline Lotação (capital) &,- 530 &, 110 &, 589 \\
\hline Idade &, 046 &, 072 & 1,047 \\
\hline Remuneração Média &, 047 &, 710 & 1,048 \\
\hline Tempo de Empresa &,- 053 &, $\mathbf{0 2 1}$ &, $\mathbf{9 4 8}$ \\
\hline Oportunidades para a aprendizagem contínua &,- 080 &, 510 &, 924 \\
\hline
\end{tabular}




\begin{tabular}{|l|c|c|c|}
\hline Questionamento e diálogo &, 169 &, 288 & 1,184 \\
\hline Colaboração e aprendizagem em equipe &, 216 &, 209 & 1,242 \\
\hline Sistemas para capturar e compartilhar a aprendizagem & $\mathbf{3 5 7}$ & $\mathbf{, 0 0 9}$ & 1,429 \\
\hline Delegação de poder e responsabilidade & $\mathbf{4 8 4}$ & $\mathbf{, 0 0 1}$ & 1,623 \\
\hline Desenvolvimento da visão sistêmica da organização & $\mathbf{4 6 0}$ & $\mathbf{, 0 0 3}$ & 1,584 \\
\hline Estímulo à liderança estratégica para a aprendizagem &, 149 &, 274 & 1,160 \\
\hline Constante & $-8,035$ &, 001 &, 000 \\
\hline
\end{tabular}

Fonte: Dados da pesquisa.

A variável que apresentou maior valor $p$ foi a que está relacionada à "Delegação de poder e responsabilidade", com valor p de 0,001 e B de 0,484. Isso significa que na percepção dos indivíduos, nessa organização, as pessoas são envolvidas em estabelecer, ter e implementar uma visão coletiva. A responsabilidade é distribuída na hora de tomar decisões para motivar as pessoas a aprender aquilo que será de sua responsabilidade, por meio da permissão das pessoas ao controle sobre os recursos de que precisam para realizar o trabalho. A variável "Delegação de poder e responsabilidade" tem uma influência de $62,3 \%$ no desempenho financeiro.

O “Desenvolvimento da visão sistêmica da organização" mostrou também influência no desempenho financeiro, com valor p de 0,003 e B de 0,460; e isto permite inferir que os indivíduos nessa organização são ajudados a ver o efeito de seu trabalho em toda a empresa. Além disso, examinam o ambiente e usam as informações para ajustar as práticas do trabalho, em conjunto com a comunidade externa. Essa variável possibilita uma influência de 58,4\% no desempenho financeiro.

Além desses achados, considera-se ainda a variável "Sistemas para capturar e compartilhar a aprendizagem", com valor $p$ de 0,009 e B de 0,357. Esta variável deteve-se influência positiva no desempenho financeiro, principalmente, na perspectiva de que os indivíduos percebem que a organização cria, integra e mantém ao trabalho sistemas de alta e baixa tecnologia para compartilhar a aprendizagem, dando acesso às pessoas às lições aprendidas. Portanto, esta variável tem uma representatividade de $42,9 \%$ no desempenho financeiro.

Em relação ao tempo de empresa, destaca-se uma influência inversa - valor $p$ de 0,021 e B de -0,53; quando maior o tempo do indivíduo na empresa, menor a sua influência no desempenho financeiro.

Nos estudos de Silva et al. (2013) e Awasthy e Gupta (2011), foram constatadas a influência das dimensões "Sistemas para capturar e compartilhar a aprendizagem" e "Desenvolvimento da visão sistêmica da organização" na "Dimensão desempenho financeiro".

A análise desta regressão logística expressa $R^{2}$ de Nagelkerke de 0,498. Esse valor indica que 
o modelo adotado é aceitável para explicar a relação das variáveis no modelo, tendo em vista $R^{2}$ de Nagelkerke ter apresentado resultado superior a 0,3.

Ao adotar o desempenho do conhecimento organizacional, realiza-se outro modelo de regressão logística, com foco no desempenho do conhecimento organizacional. A Tabela 6 retrata o modelo de regressão para o desempenho do conhecimento organizacional (variável dependente) na cultura de aprendizagem, bem como nos dados sociodemográficos (variáveis independentes).

Tabela 6 - Modelo de regressão

(variável dependente: desempenho do conhecimento organizacional)

\begin{tabular}{|c|c|c|c|}
\hline Variáveis & SB & Sig. & $\operatorname{Exp}(B)$ \\
\hline Sexo (Masculino) & ,863 & ,004 & 2,369 \\
\hline Escolaridade (Médio/Técnico) & 2,246 & 259 & 9,448 \\
\hline Escolaridade (Graduação) & 2,426 & 217 & 11,319 \\
\hline Escolaridade (Especialização) & 1,976 & ,308 & 7,210 \\
\hline Escolaridade (Mestrado) & 1,753 & ,374 & 5,771 \\
\hline Lotação (capital) &,- 291 & ,402 & ,747 \\
\hline Sexo (Masculino) &,- 011 & 675 & 989 \\
\hline Idade &,- 001 & 996 & ,999 \\
\hline Remuneração Média & ,004 & ,872 & 1,004 \\
\hline Tempo de Empresa &,- 111 & 375 & ,895 \\
\hline Oportunidades para a aprendizagem contínua &,- 146 & ,406 & ,864 \\
\hline Questionamento e diálogo & 2,246 & ,259 & 9,448 \\
\hline Colaboração e aprendizagem em equipe & ,664 & ,000 & 1,942 \\
\hline Sistemas para capturar e compartilhar a aprendizagem & ,335 & ,022 & 1,398 \\
\hline Delegação de poder e responsabilidade & ,323 & 035 & 1,382 \\
\hline Desenvolvimento da visão sistêmica da organização & ,625 & ,000 & 1,869 \\
\hline Estímulo à liderança estratégica para a aprendizagem & ,346 & ,015 & 1,413 \\
\hline Constante & $-8,816$ & ,000 & ,000 \\
\hline
\end{tabular}

Fonte: Dados da pesquisa.

A Colaboração e aprendizagem em equipe" apresenta influência na dimensão "Desempenho do conhecimento organizacional", com valor $p$ de 0,000 e B de 0,664. O indivíduo que enfatizou a percepção da colaboração e aprendizagem em equipe tem $94,2 \%$ de chance de ter a percepção do desempenho do conhecimento organizacional.

Outra variável com alto poder de explicação na dimensão "Desempenho do conhecimento organizacional" é a "Desenvolvimento da visão sistêmica da organização", com valor p de 0,000 e B de 0,625 . Desse modo, identifica-se influência positiva no "Desenvolvimento da visão sistêmica da organização" no "Desempenho do conhecimento organizacional". 
Além dessas, outras variáveis que tiveram influência positiva no desempenho do conhecimento organizacional foram "Estímulo à liderança estratégica para a aprendizagem", com valor p de 0,015 e B de 0,346; "Sistemas para capturar e compartilhar a aprendizagem", com valor p de 0,022 e B de 0,335; e "Delegação de poder e responsabilidade", com valor p de 0,035 e B de 0,323. Essas variáveis tiveram influência positiva no desempenho do conhecimento organizacional.

Ressalta-se a perspectiva de Schein (2009) ao afirmar que o líder ter a compreensão da complexidade do mundo o qual está interconectado, tomando-se como base a influência da liderança no desempenho do conhecimento organizacional.

Em sua pesquisa Kumar et al. (2016), observaram que, mesmo em um país em desenvolvimento como a Índia, que progrediu em vários setores da economia, não apresentava o mesmo progresso na área da saúde. Com o desenvolvimento de uma cultura de aprendizagem nas empresas de saúde da Índia, foi possível observar progressos nas metas de desenvolvimento do milênio.

Os estudos de Silva et al. (2013), também apontaram que a cultura de aprendizagem possui forte influência no desempenho organizacional quando essa relação foi pesquisada em emissoras de TV cearenses.

Os resultados apresentados alinham-se com os resultados encontrados por Awasthy e Gupta (2011) em sua pesquisa, quando as dimensões que obtiveram maior poder explicativo no desempenho do conhecimento organizacional foram as mesmas encontradas nesta pesquisa.

A análise desta regressão logística expressa $R^{2}$ de Nagelkerke de 0,552. Esse valor indica que o modelo adotado é aceitável para explicar a relação das variáveis no modelo, tendo em vista $R^{2}$ de Nagelkerke ter apresentado resultado superior a 0,3.

Ao considerar que o desempenho financeiro e o desempenho do conhecimento organizacional pertencem ao desempenho organizacional, identifica-se, sobretudo, que dentre os 7 fatores que representam a aprendizagem organizacional, 3 mostram-se alinhados tanto com o financeiro e o desempenho do conhecimento organizacional. De modo geral, esses 3 fatores -Sistemas para capturar e compartilhar a aprendizagem, Delegação de poder e responsabilidade e Desenvolvimento da visão sistêmica da organização tiveram influência positiva no desempenho organizacional.

Desta forma, os resultados corroboram com o que defendem para Marsick e Watkins (2003), quando afirmam que a cultura de aprendizagem exerce significativa influência na melhoria do conhecimento organizacional e financeiro. 


\section{CONCLUSÃO}

A aprendizagem surgiu como facilitadora do processo de adequação às mudanças do ambiente para as organizações, levando em consideração o conjunto dos valores organizacionais, as relações estabelecidas pelos diversos atores da organização e a coletividade envolvida no processo de aprendizagem dentro da organização (QUEIROZ et al., 2014).

Quando nos referimos ao desenvolvimento organizacional, considerando aspectos como o desempenho financeiro e o desempenho do conhecimento, invariavelmente temos como elementos influenciadores, aqueles relacionados à cultura, especificamente à cultura de aprendizagem, tendo em vista que a cada dia, esses elementos da cultura são utilizados de forma a aumentar o desempenho organizacional.

Nesse sentido, esse estudo teve como objetivo analisar a influência da cultura de aprendizagem no desempenho organizacional.

Para realização deste estudo, foi realizada pesquisa com os colaboradores da empresa, para, em seguida, ser realizada as análises estatísticas necessárias para verificação das relações de influência existentes entre os construtos cultura de aprendizagem e desempenho organizacional.

Conforme apontado pelos resultados, a cultura de aprendizagem exerce forte influência no desempenho organizacional na empresa ALFA, por meio da percepção dos respondentes.

As dimensões da cultura de aprendizagem que mais influenciam o desempenho organizacional são "Colaboração e aprendizagem em equipe" e "Delegação de poder e responsabilidade". No caso da dimensão "Colaboração e aprendizagem em equipe", esta apresenta forte influência na dimensão do desempenho organizacional "Desempenho do conhecimento". Já a dimensão "Delegação de poder e responsabilidade", exerce forte influência na dimensão "Desempenho financeiro" do construto "Desempenho organizacional".

A partir desses resultados, diversas estratégias podem traçadas com relação aos estudos na área de gestão de pessoas. Como relevância para a gestão, espera-se que os resultados desta pesquisa colaborem para a formulação de políticas de gestão de pessoas no que se refere ao desenvolvimento da liderança de forma a permitir a geração de desempenho positivo. Ainda como contribuição, no âmbito acadêmico, este estudo colabora para diminuir a lacuna existente na literatura que permita analisar as influências entre a cultura de aprendizagem e o desempenho organizacional, no que se refere à investigação em empresa do setor público, bem como permite realizar comparações entre esses resultados com os resultados em empresas privadas.

Considerando a relevância da temática, apontam-se como agenda de pesquisa a necessidade de expandir para outras organizações, inclusive em outros setores da economia, estudos que veri- 
fiquem as influências da cultura de aprendizagem no desempenho organizacional. Além disso, na mesma empresa, este estudo possibilita pesquisas com empregados terceirizados a fim de comparar com os resultados deste estudo, já que esta organização apresenta grande número dessa categoria de empregados.

\section{REFERÊNCIAS BIBLIOGRÁFICAS}

ARRUDA, S. C. et al. Cultura de aprendizagem e desempenho organizacional em OSCIPS. Revista Pretexto, v. 16, n. 2, p. 98-116, 2015.

AWASTHY, R.; GUPTA, R. K. Is learning orientation in manufacturing and service firms different in India? The learning organization, v. 18, n. 5, p. 392-408, 2011.

AZANZA, G.; MORIANO, J. A.; MOLERO, F. Authentic leadership and organizational culture as drivers of employees' job satisfaction. Journal of Work and Organizational Psychology. 29, p. 4550, 2013.

\section{BABBIE, E. Métodos de pesquisas de survey, 3. ed. Belo Horizonte: UFMG, 2005.}

BATES, R.; KHASAWNEH, S. Organizational learning culture, learning transfer climate and perceived innovation in Jordanian organizations. International journal of training and development, v. $9, n$. 2, p. 96-109, 2005.

BRANDÃO, H. P.; BORGES-ANDRADE, J. E.; GUIMARÃES, T A. Desempenho organizacional e suas relações com competências gerenciais, suporte organizacional e treinamento. Revista de Administração, v. 47, n. 4, p. 523-539, 2012.

BRITO, L. A. L.; VASCONCELOS, F. C. Desempenho das empresas brasileiras: efeitos ano, ramo de negócios e firma individual. Revista de Administração Contemporânea, p. 65, 2005.

BATES, R.; KHASAWNEH, S. Organizational learning culture, learning transfer climate and perceived innovation in Jordanian organizations. International journal of training and development, v. $9, n$. 2, p. 96-109, 2005.

COLLIS, J.; HUSSEY, R. Pesquisa em administração: um guia prático para alunos de graduação e pós-graduação. Porto Alegre: Bookman, 2005.

DIAS, R. Cultura organizacional: construção, consolidação e mudanças. São Paulo: Atlas, 2013.

DELANEY, J. T.; HUSELID, M.A. The impact of human resource management practices on perceptions of organizational performance. Academy of Management journal, v. 39, n. 4, p. 949-969, 1996.

DESHPANDE, R.; WEBSTER, F. E. Organizational culture and marketing: defining the research agenda. The journal of marketing, p. 3-15, 1989.

FERNANDES, A. L.; ALTURAS, B. A. B.; LAUREANO, R. M. S. The role of organizational culture in the dynamic relationship betwenn information Technology and organizational learning. In: Information Systems and Technologies (CISTI), 2015 10th Iberian Conference on. IEEE, p. 1-5, 2015.

FERREIRA, M. C.; ASSMAR, E. M. L. Cultura organizacional. In: SIQUEIRA, Mirlene Maria Matias. Medidas do comportamento organizacional: ferramentas de diagnóstico e de gestão. Porto Alegre: Artmed, 2008.

FIELD, A. Descobrindo a estatística usando o SPSS-2. Bookman Editora, 2009. 
FIOL, C. Marlene; LYLES, Marjorie A. Organizational learning. Academy of management review, v. 10, n. 4, p. 803-813, 1985.

FORTADO, B.; FADIL, P. The four faces of organizational culture. Competitiveness Review: an international business journal, v. 22, n. 4, p. 283-298, 2012.

HAIR, J. F.; BLACK, W. C.; BABIN, B. J.; ANDERSON, R. E.; TATHAM, R. L. Análise Multivariada de Dados. Tradução: Adonai Schlup Sant'anna. 6. ed. Porto Alegre: Bookman, 2009.

HUNG, R. Y. Y. et al. Dynamic capability: Impact of process alignment and organizational learning culture on performance. Journal of World Business, v. 45, n. 3, p. 285-294, 2010.

HOGAN, S. J.; COOTE, L. V. Organizational culture, innovation, and performance: A test of Schein's model. Journal of Business Research, v. 67, n. 8, p. 1609-1621, 2014.

KAPLAN, R. S.; NORTON, D. P. Mapas estratégicos: convertendo ativos intangíveis em resultados tangíveis. Gulf Professional Publishing, 2004.

KUMAR, J. K. et al. An Insight into Health Care Setup in National Capital Region of India using Dimensions of Learning Organizations Questionnaire (DLOQ)-A Cross-Sectional Study. Journal of Clinical and Diagnostic Research: JCDR, v. 10, n. 6, p. ZC01, 2016.

JUNG, T. et al. Instruments for exploring organizational culture: A review of the literature. Public administration review, v. 69, n. 6, p. 1087-1096, 2009.

LAU, P. Y. Y. et al. Learning organization, organizational culture, and affective commitment in Malaysia: A person-organization fit theory. Human Resource Development International, v. 20, n. 2, p. 159179, 2017.

LOIOLA, E.; LEOPOLDINO, C. Aprendizagem organizacional e desempenho: o que podemos aprender dessa relação? Revista Psicologia Organizações e Trabalho, v. 13, n. 2, p. 187-201, 2013.

MARANHÃO, M. L.; DE SÁ, M. A. D. Cultura organizacional e práticas de qualidade de vida no trabalho: um estudo de múltiplos casos em palcos organizacionais de restaurantes do Recife-PE. Revista de Carreiras e Pessoas (ReCaPe). ISSN 2237-1427, v. 9, n. 2, p. 267-286, 2019.

MARSICK, V. J.; WATKINS, K. E. Demonstrating the value of an organization's learning culture: the dimensions of the learning organization questionnaire. Advances in developing human resources, v. 5, n. 2, p. 132-151, 2003.

MENEZES, E. A. C.; GUIMARÃES, T. A.; BIDO, D. S. Dimensões da aprendizagem em organizações: validação do Dimensions of the Learning Organization Questionnaire (DLOQ) no contexto brasileiro. Revista de Administração Mackenzie, v. 12, n. 2, p. 4, 2011.

MORGAN, G. Imagens da organização. 1. ed. São Paulo: Atlas, 2010.

NICA, E. a et al. Organizational culture in the public sector. Economics, Management, and Financial Markets, v. 8, n. 2, p. 179-184, 2013.

OLIVA, C. C.; SHINYASHIKI, G. T. Estudo sobre validação de escalas de aprendizagem organizacional no Brasil. Revista Brasileira de Gestão e Desenvolvimento Regional, v. 12, n. 3, 2016.

QUEIROZ, G. C., et al. Cultura de aprendizagem e privatização: um estudo de caso na Transnordestina. Reuna. Belo Horizonte, v. 19, n. 3, p. 29-48, 2014.

RAFAELI, L.; MÜLLER, C. J. Estruturação de um índice consolidado de desempenho utilizando o AHP. Gestão \& Produção, v. 14, n. 2, p. 363-377, 2007. 
RICHARD, P. J. et al. Measuring organizational performance: Towards methodological best practice. Journal of management, v. 35, n. 3, p. 718-804, 2009.

RIJAL, S. The influence of transformational leadership and organizational culture on learning organization: A comparative analysis of the it sector. Journal of Administrative and Business studies, v. 2, n. 3, 2016.

ROBBINS, S. P. Fundamentos do comportamento organizacional. São Paulo: Pearson Prentice Hall, 2009.

SCHEIN, E. H. Organizational culture and leadership. San Francisco: JosseyBass, 2009.

SCHMITZ, S.; et al. Learning culture and knowledge management processes: to what extent are they effectively related? Journal of Work and Organizational Psychology. v 30, n. 3, p. 113-121, 2014.

SENGE, P. M. The fifth discipline: The art and practice of the learning organization. Crown Pub, 2006.

SILVA, A. et al. Cultura de aprendizagem e desempenho nas TVs cearenses. R. Adm. FACES Journal Belo Horizonte. v. 12, n. 4, p. 30-48, 2013.

STEWART, T. A. A riqueza do conhecimento: o capital intelectual e a organização do século XXI. Rio de Janeiro: Campus, 2002.

VARGAS-HERNÁNDEZ, J. et al. Performance y aprendizaje organizacional bajo el enfoque de las teorías organizacionales. Perspectiva Socioeconómica, v. 1, n. 3, p. 5-16, 2016.

WOOD JR.; T. Comportamento organizacional: uma perspectiva brasileira. 2 ed. São Paulo: Atlas, 2007.

YANG, B. Identifying valid and reliable measures for dimensions of a learning culture. Advances in developing human resources, v. 5, n. 2, p. 152-162, 2003. 